\title{
GENERALIZED LEAST SQUARES ESTIMATORS FOR RANDOMIZED FRACTIONAL REPLICATION DESIGNS ${ }^{1}$
}

\author{
By S. ZACKS \\ Technion, Israel Institute of Technology and Stanford University
}

1. Introduction. The problem of choosing a $1 / M=1 / 2^{m-s}(m>s)$ fractional replication from a factorial system of size $N=2^{m}$ and deciding upon appropriate estimator of the parameters characterizing the factorial model (main effects and interactions) has been studied by Dempster [2], [3], Takeuchi [11], [12], Ehrenfeld and Zacks [4], [5], Zacks [13], Shah and Kempthorne [9], [10]. In all these studies the type of estimators considered is that which yields, under a randomized procedure with equal probabilities of choice, unbiased estimates of a specified subvector of parameters, which lies in the range of the design matrix. A complete class of linear unbiased estimators for estimating an $M$-dimensional subvector of pre-assigned parameters, under a randomized fractional replication procedure, was studied in [13]. Optimal procedures of choosing a fractional replication and an estimator were studied in [5].

In the present paper we consider the problem of estimating the entire vector of $N$ parameters, on the basis of a fractional replication of size $S=2^{s}$. This problem arises when we wish to explore a given factorial system, and find which are the important parameters. Such a problem calls for a sequence of fractional replication designs, where each design is modified by the information attained in previous experiments. At least in the first stages of such a sequence one would like to estimate the entire vector of $N$ parameters. Presently we consider this estimation problem for a one stage fractional replication design. We adopt the least-squares principle, and consider the class of all generalized least-squares estimators (g.l.s.e.) which corresponds to a given block of $S$ treatment combinations. The term generalized least-squares estimators is used since the corresponding matrices of the normal equations are singular. The linear spaces of all g.l.s.e. associated with the various fractional replication designs, of the type considered here (see Ehrenfeld and Zacks [4] and Zacks [13]), are characterized in terms of the linear coefficients of the factorial model. As proven, every g.l.s.e. is represented by an $M$-dimensional vector $\left(\lambda_{0}, \cdots, \lambda_{M-1}\right)$ where $\sum_{u=0}^{M-1} \lambda_{u}=1$. Some statistical properties of the g.l.s.e., under randomized fractional replication, are studied. First we prove that there is no randomized fractional replication procedure for which an unbiased g.l.s.e. of the entire vector of parameters exists. The problem of which fractional replication to use and which g.l.s.e. to apply is studied in a general decision framework, with the trace of the mean-square-error

Received 2 May 1963.

1 This work was supported in part by Office of Naval Research Contract Nonr-225(52) at Stanford University. Reproduction in whole or in part is permitted for any use of the United States Government. 
matrix as a risk function. As proven, when the parameters of the factorial model may assume arbitrary values, the randomization procedure which assigns equal probabilities to various fractional replications (denoted by R.P.*) is the only admissible procedure for that risk function. Bayes g.l.s.e. for a prior information available on the parameters are studied. This leads to a minimax theorem, which specifies a minimax and admissible g.l.s.e. under R.P.* Finally, by applying a theorem of Rao [8] we characterize the class of all functionals which yield estimable linear functions of the parameters, and which have uniformly minimum variance g.l.s.e.

2. The factorial model and randomization procedures. The factorial model and randomization procedures are presented in the present section. To save space, the reader is referred to [4] and [13] for details and definitions of terms used here. Familiarity with the algebra of factorial experiments is assumed (see [7]).

Consider factorial systems of order $N=2^{m}$, and fractional replications of order $S=2^{s}(1 \leqq s<m)$. Let $X_{v}(v=0, \cdots, M-1)$ designate a block of $S$ treatment combinations. To simplify the presentation of the results, we assume that the blocks $X_{v}$ are constructed by assigning to the $m-s$ "main effects" $\left\{\beta_{S}, \beta_{2 S}, \cdots, \beta_{N-S}\right\}$ the role of independent defining parameters. The general presentation can be easily carried out, as shown in [4]. Relative to this set of defining parameters, the $M$ blocks of treatment combinations are:

$$
\begin{aligned}
& X_{v}=\left(\mathrm{x}: \text { if } \mathrm{x} \equiv\left(i_{0}, \cdots, i_{m-1}\right)\right. \text { and } \\
& \left.\qquad t=\sum_{j=0}^{m-1} i_{j} 2^{j} \text { then } t \equiv v(\bmod . M)\right) .
\end{aligned}
$$

In other words, $X_{v}$ contains all the treatment combinations x whose standard order is equivalent to $v$ modulo $M$. Here $i_{j}=0,1$ denotes the level of factor $j(j=0, \cdots, m-1)$. Let $Y\left(X_{v}\right)$ be a random vector of order $S$, representing the yield of the treatment combinations in block $X_{v}$. Let $\beta=\left(\beta_{0}, \cdots, \beta_{N-1}\right)^{\prime}$ be a parameter vector of the factorial system, representing main effects and interactions. The relationship between $Y\left(X_{v}\right)$ and $\beta$ is given by the linear model of orthogonal contrasts

$$
Y\left(X_{v}\right)=\left(C_{v}\right) \beta+\epsilon, \quad v=0, \cdots, M-1
$$

where $\epsilon$ is a random vector representing the experimental errors, having $E\{\epsilon\}=0$ and $E\left\{\epsilon \epsilon^{\prime}\right\}=\sigma^{2} I^{(S)}$. $\left(C_{v}\right)$ is a $S \times N$ matrix, which is the $v$ th submatrix of the matrix $\left(C^{(N)}\right)$, corresponding to the full factorial system. The rectangular matrix $\left(C_{v}\right)$ is related to the lower order matrices $\left(C^{(S)}\right)$ and $\left(C^{(M)}\right)$ according to

$$
\left(C_{v}\right)=\left(1, c_{v 1}^{(M)}, \cdots, c_{v, M-1}^{(M)}\right) \otimes\left(C^{(S)}\right), \quad v=0, \cdots, M-1
$$

where $c_{v u}^{(M)}(v, u=0, \cdots, M-1)$ are the elements of $\left(C^{(M)}\right) ;\left(C^{(N)}\right)=\left(C^{(M)}\right)$ $\otimes\left(C^{(S)}\right)$, and for each $k=2,3, \cdots$ 


$$
\left(C^{\left(2^{k}\right)}\right)=\left(C^{(2)}\right) \otimes\left(C^{\left(2^{k-1}\right)}\right), \text { where } \quad\left(C^{(2)}\right)=\left[\begin{array}{l}
1-1 \\
1-1
\end{array}\right] .
$$

$\otimes$ denotes the Kroneker's direct multiplication operator. According to (2.2) and (2.3) we can write the factorial model in the form:

$$
Y\left(X_{v}\right)=\sum_{u=0}^{M-1} c_{v u}^{(M)}\left(C^{(S)}\right) \beta_{(u)}+\epsilon, \quad v=0, \cdots, M-1
$$

where $\beta_{(u)}^{\prime}=\left(\beta_{u s}, \beta_{u S+1}, \cdots, \beta_{(u+1) S-1}\right)$ is the $u$ th subvector of $\beta$.

A randomized fractional replication procedure is one in which each block $X_{v}(v=$ $0, \cdots, M-1)$ is chosen with probability $\xi_{v}\left(\xi_{v} \geqq 0 ; \sum_{v=0}^{M-1} \xi_{v}=1\right)$. Every randomized fractional replication procedure is represented by an $M$-dimensional probability vector $\xi$. This class of randomized procedures contains in particular the fixed fractional replication designs, in which one of the blocks is chosen with probability one.

3. Generalized least squares estimators for fractional replications. Consider a block $X_{v}(v=0, \cdots, M-1)$ of treatment combinations. We shall characterize now the class of all g.l.s.e. corresponding to $X_{v}$. Following the least-squares principle, the "normal equations" corresponding to the factorial model (2.2) are:

$$
\left(C_{v}\right)^{\prime}\left(C_{v}\right) \hat{\beta}_{v}=\left(C_{v}\right)^{\prime} Y\left(X_{v}\right)
$$

$\hat{\beta}_{v}$ designates a generalized least-square estimate of $\beta$.

A generalized least-square estimator (g.l.s.e.) of $\beta$ is any linear operator $(L)$ on $E^{(S)}$ (Euclidean $S$-space) such that $\left(L_{v}\right)$ is an $N \times S$ matrix satisfying the equation:

$$
\left(C_{v}\right)^{\prime}\left(C_{v}\right)\left(L_{v}\right)=\left(C_{v}\right)^{\prime} \text {. }
$$

Decompose $\left(L_{v}\right)$ into $M$ submatrices $\left(L_{v u}\right)$ of order $S \times S$, i.e., $\left(L_{v}\right)^{\prime}=\left(\left(L_{v 0}\right)^{\prime}\right.$, $\left.\cdots,\left(L_{v(M-1)}\right)^{\prime}\right)$. Since $\left(C^{(S)}\right)^{\prime}\left(C^{(S)}\right)=S I^{(S)}$ it follows from (2.3) that (3.2) is equivalent to

$$
\left.S\left[Q^{(M)}\right) \otimes I^{(S)}\right]\left[\begin{array}{l}
\left(L_{v 0}\right) \\
\vdots \\
\left(L_{v(M-1)}\right)
\end{array}\right]=\left[\begin{array}{l}
1 \\
c_{v 1}^{(M)} \\
\vdots \\
c_{v(M-1)}^{(M)}
\end{array}\right] \otimes\left(C^{(S)}\right)
$$

where $\left(Q^{(M)}\right)=\left(1, c_{v 1}^{(M)}, \cdots, c_{v(M-1)}^{(M)}\right)^{\prime}\left(1, c_{v 1}^{(M)}, \cdots, c_{v(m-1)}^{(M)}\right)$ is a square matrix of order $M \times M$. Furthermore, since $c_{v u}^{(M)}= \pm 1$, Equation (3.3) can be represented by the following equation:

$$
\sum_{u=0}^{M-1} c_{v u}^{(M)}\left(L_{v u}\right)\left(C^{(S)}\right)=\left(I^{(S)}\right)
$$

Consider the $M$ matrices $\left(L_{v}^{(j)}\right), j=0, \cdots, M-1$, whose submatrices are given by: 


$$
\begin{aligned}
\left(L_{v u}^{(j)}\right) & =S^{-1} c_{v u}^{(M)}\left(C^{(S)}\right)^{\prime}, \\
& =(0),
\end{aligned}
$$

if $j=u$

otherwise.

These matrices satisfy Equation (3.4) and thus constitute a basis of $M$ independent solutions to (3.2). Every solution to (3.2) can be represented as a linear combination of the $M$ linearly independent operators $\left(L_{v}^{(j)}\right)$, namely

$$
\left(L_{v}\right)=\sum_{j=0}^{M-1} \lambda_{j}\left(L_{v}^{(j)}\right) \quad \text { where } \quad \sum_{j=0}^{M-1} \lambda_{j}=1 .
$$

Thus, the class of all g.l.s.e. corresponding to a given fractional replication $X_{v}(v=0, \cdots, M-1)$ is

$$
\mathscr{L}\left(X_{v}\right)=\left(\left(L_{v}\right):\left(L_{v}\right)=\sum_{j=0}^{M-1} \lambda_{j}\left(L_{v}^{(j)}\right) ; \sum_{j=0}^{M-1} \lambda_{j}=1\right) .
$$

Accordingly, every g.l.s.e. can be represented by an $M$-dimensional real vector $\lambda^{\prime}=\left(\lambda_{0}, \cdots, \lambda_{M-1}\right)$ such that $\sum_{j=0}^{M-1} \lambda_{j}=1$. Moreover, since $\hat{\beta}_{v}=\left(L_{v}\right) Y\left(X_{v}\right)$ we have,

$$
\hat{\beta}_{v}=S^{-1}\left(\lambda_{0}, \lambda_{1} c_{v 1}^{(M)}, \cdots, \lambda_{M-1} c_{v(M-1)}^{(M)}\right)^{\prime} \otimes\left(C^{(S)}\right)^{\prime} Y\left(X_{v}\right) .
$$

4. Some statistical properties of g.l.s.e. In the present section we prove that there are no unbiased g.l.s.e. of the entire vector of parameters $\beta$, and derive an expression for the trace of the mean-square-error matrix of a g.l.s.e.

THEOREM 1. There is no unbiased g.l.s.e. of $\beta$

Proof. Let $\tilde{\beta}_{(u)}=S^{-1}\left(C^{(S)}\right)^{\prime} Y\left(X_{v}\right), u=0, \cdots, M-1$. As proven in

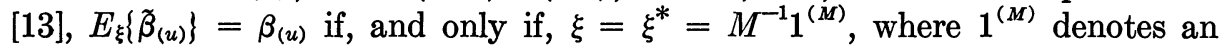
$M$-dimensional vector whose components are all equal to one. Accordingly

$$
\begin{aligned}
E_{\xi^{*}}\left\{\hat{\beta}^{\prime}\right\}=E_{\xi^{*}}\left\{\left(\lambda_{0} \widetilde{\beta}_{(0)}^{\prime}, \lambda_{1} \widetilde{\beta}_{(1)}^{\prime}, \cdots, \lambda_{M-1} \widetilde{\beta}_{M-1}^{\prime}\right)\right\} & \\
& =\left(\lambda_{0} \beta_{(0)}^{\prime}, \cdots, \lambda_{M-1} \beta_{(M-1)}^{\prime}\right) .
\end{aligned}
$$

Since $\sum_{j=0}^{M-1} \lambda_{j}=1$ the theorem is proven.

The g.l.s.e. in which $\lambda_{0}=1$ and $\lambda_{u}=0$ for all $u>0$ yields unbiased estimates of the components of $\beta_{(0)}$ only. Similarly when $\lambda_{j}=1(j=0, \cdots, M-1)$ and $\lambda_{j^{\prime}}=0$ for all $j^{\prime} \neq j$, the corresponding g.l.s.e. yields unbiased estimates of the components of $\beta_{(j)}$ only.

The mean-square-error dispersion matrix of a g.l.s.e. $\hat{\beta}$, under randomization procedure $\xi$, is defined by $E_{\xi}\left\{(\hat{\beta}-\hat{\beta})(\hat{\beta}-\beta)^{\prime}\right\}$. Let $M(\xi, \lambda ; \beta)$ denote the trace of the mean-square-error dispersion matrix of a g.l.s.e. represented by a vector $\lambda$, such that $\lambda^{\prime} 1^{(M)}=1$, under randomization procedure $\xi$, i.e., $M(\xi, \lambda ; \beta)=$ $E_{\xi}\left\{(\hat{\beta}-\beta)^{\prime}(\hat{\beta}-\beta)\right\}$.

THEOREM 2. The trace of the mean-square-error dispersion matrix under randomization procedure $\xi$ is given by:

$$
\begin{aligned}
M(\xi, \lambda ; \beta)=\left(\sigma^{2}\right. & \left.+|\beta|^{2}\right) \sum_{u=0}^{M-1} \lambda_{u}^{2}-\sum_{u=0}^{M-1}\left(2 \lambda_{u}-1\right)\left|\beta_{(u)}\right|^{2} \\
& +\sum_{u_{1}=0}^{M-1}\left(2 \lambda_{u_{1}}+1\right) \sum_{\left(u_{2} \neq u_{1}\right)=0}^{M-1} \sum_{v=0}^{M-1} \xi_{v} c_{\left.v u_{1}\right)}^{(M)} c_{v u_{2}}^{(M)} \beta_{\left(u_{1}\right)}^{\prime} \beta_{\left(u_{2}\right)}
\end{aligned}
$$


where $|\beta|^{2}=\beta^{\prime} \beta,\left|\beta_{(u)}\right|^{2}=\beta_{(u)}^{\prime} \beta_{(u)}(u=0, \cdots, M-1)$.

Proof. By definition,

$$
M(\xi, \lambda ; \beta)=E_{\xi}\left\{(\hat{\beta}-\beta)^{\prime}(\hat{\beta}-\beta)\right\}=E_{\xi}\left\{\hat{\beta}^{\prime} \hat{\beta}\right\}-2 \beta^{\prime} E_{\xi}\{\hat{\beta}\}+\beta^{\prime} \beta .
$$

According to (3.8)

$$
E_{\xi}\left\{\hat{\beta}^{\prime} \hat{\beta}\right\}=S^{-1} \sum_{u=0}^{M-1} \lambda_{u}^{2} E_{\xi}\left\{Y\left(X_{v}\right)^{\prime} Y\left(X_{v}\right)\right\}
$$

Substituting (2.5) for $Y\left(X_{v}\right)$ in (4.4) we get:

$$
\begin{aligned}
& S^{-1} E_{\xi}\left\{Y\left(X_{v}\right)^{\prime} Y\left(X_{v}\right)\right\}=S^{-1} E_{\xi}\left\{\left[\sum_{u_{1}=0}^{M-1} c_{v u_{1}}^{(M)}\left(C^{(S)}\right) \beta_{\left(u_{1}\right)}+\epsilon\right]^{\prime}\right. \\
& \left.\cdot\left[\sum_{u_{2}=0}^{M-1} c_{v u_{2}}^{(M)}\left(C^{(S)}\right) \beta_{\left(u_{2}\right)}+\epsilon\right]\right\} \\
& =\sigma^{2}+S^{-1} E_{\xi}\left\{\sum_{u_{1}=0}^{M-1} \sum_{u_{2}=0}^{M-1} c_{v u_{1}}^{(M)} c_{v u_{2}}^{(M)} \beta_{\left(u_{1}\right)}^{\prime}\left(C^{(S)}\right)^{\prime}\left(C^{(S)}\right) \beta_{\left(u_{2}\right)}\right\} \\
& =\sigma^{2}+\sum_{u=0}^{M-1}\left|\beta_{(u)}\right|^{2}+\sum_{u_{1} \neq u_{2}} \sum_{v=0}\left(\sum_{v-1}^{M-1} \xi_{v} c_{v u_{1}}^{(M)} c_{v u_{2}}^{(M)} \beta_{\left(u_{1}\right)}^{\prime} \beta_{\left(u_{2}\right)}\right) .
\end{aligned}
$$

Furthermore, $E_{\xi}\left\{\hat{\beta}^{\prime}\right\}=\left(\lambda_{0} E_{\xi}\left\{\tilde{\beta}_{(0)}^{\prime}\right\}, \cdots, \lambda_{M-1} E_{\xi}\left\{\tilde{\beta}_{(M-1)}^{\prime}\right\}\right)$, where

$$
E_{\xi}\left\{\tilde{\beta}_{(u)}\right\}=\beta_{(u)}+\sum_{(w \neq u)=0}^{M-1} \sum_{v=0}^{M-1} \xi_{v} c_{v u}^{(M)} c_{v u}^{(M)} \beta_{(w)} .
$$

Hence,

$$
\begin{aligned}
\beta^{\prime} E_{\xi}\{\hat{\beta}\}=\sum_{u=0}^{M-1} \lambda_{u}\left|\beta_{(u)}\right|^{2} & \\
& +\sum_{u_{1}=0}^{M-1} \lambda_{u}\left[\sum_{u_{2} \neq u_{1}}\left(\sum_{v=0}^{M-1} \xi_{v} c_{v u_{1}}^{(M)} c_{v u_{2}}^{(M)}\right) \cdot \beta_{\left(u_{1}\right)}^{\prime} \beta_{\left(u_{2}\right)}\right] .
\end{aligned}
$$

Thus, from (4.3)-(4.7) the result holds.

Corollary 2.1. When each block $X_{v}(v=0, \cdots, M-1)$ is chosen with equal probabilities $\left(\xi=\xi^{*}\right)$ then

$$
M\left(\xi^{*}, \lambda ; \beta\right)=\left(\sigma^{2}+|\beta|^{2}\right) \sum_{u=0}^{M-1} \lambda_{u}^{2}-\sum_{u=0}^{M-1}\left(2 \lambda_{u}-1\right)\left|\beta_{(u)}\right|^{2} .
$$

5. Optimum strategies. A strategy of the Statistician is a pair of two $M$ dimensional vectors $(\xi, \lambda)$ such that $\xi$ is a probability vector, and $\lambda^{\prime} 1^{(M)}=1$. Every strategy $(\xi, \lambda)$ represents a randomization procedure and a g.l.s.e. The decision problem is to choose $(\xi, \lambda)$ optimally, with respect to the loss function $M(\xi, \lambda ; \beta)$.

THEOREM 3. If $\beta$ is arbitrary then $\xi^{*}$ represents the only admissible randomization procedure, relative to the loss function $M(\xi, \lambda ; \beta)$.

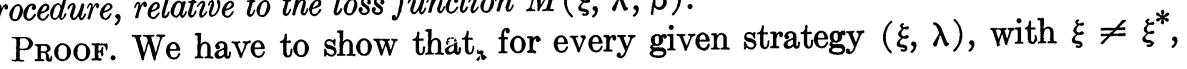


there exists $\beta^{0}$ in $E^{(N)}$ such that: $M\left(\xi, \lambda ; \beta^{0}\right)>M\left(\xi^{*}, \lambda ; \beta^{0}\right)$. Comparing (4.2) and (4.8) the problem reduces to that of finding $\beta^{0}$ such that, for the given pair $(\xi, \lambda), \xi \neq \xi^{*}$,

$$
\sum_{u_{1}=0}^{M-1}\left(2 \lambda_{u_{1}}+1\right) \sum_{\left(u_{2} \neq u_{1}\right)=0}^{M-1}\left[\sum_{v=0}^{M-1} \xi_{v} c_{v u_{1}}^{(M)} c_{v u_{2}}^{(M)}\right] \beta_{\left(u_{1}\right)}^{\prime \prime} \beta_{\left(u_{2}\right)}^{0}>0 .
$$

Without loss of generality, assume $\lambda_{0}>0$. Since $\xi \neq \xi^{*}, \sum_{v=0}^{M-1} \xi_{v} c_{v u}^{(M)} \neq 0$ for all $u \neq 0\left(c_{v 0}^{(M)}=1\right.$ for all $\left.v=0, \cdots, M-1\right)$. Let $\beta^{0}=\left(\beta_{(0)}^{0}, \beta_{(1)}^{0}, 0, \cdots, 0\right)^{\prime}$ with $\left|\beta_{(0)}^{0}\right|=1,\left|\beta_{(1)}^{0}\right|=1$ and such that the angle between the two $S$-dimensional vectors $\beta_{(0)}^{0}$ and $\beta_{(1)}^{0}, \varphi$ say, satisfies the equality:

$$
\cos \varphi=\beta_{(0)}^{0 \prime} \beta_{(1)}^{0 \prime}=\sum_{v=0}^{M-1} \xi_{v} c_{v 1}^{(M)}
$$

Then $\beta^{0}$ satisfies the inequality (5.1). This completes the proof.

Due to Theorem 3 we restrict the discussion in the sequel to strategies of the form $\left(\xi^{*}, \lambda\right)$.

We notice in (4.8) that $M\left(\xi^{*}, \lambda ; \beta\right)$ depends on $\beta$ only through the $M$ values $\left|\beta_{(u)}\right|^{2}$. An a-priori information concerning these values might thus be utilized for the choice of $\lambda$. Thus, let $\pi^{(u)}$ be an a-priori distribution of $\left|\beta_{(u)}\right|^{2}$, defined over the half-line $[0, \infty)$.

Theorem 4. The Bayes g.l.s.e. of $\beta$, with respect to the a-priori distributions $\left\{\pi^{(0)}, \cdots, \pi^{(M-1)}\right\}$, under randomization procedure $\xi^{*}$ is determined by the vector $\lambda_{\pi}=\left(\lambda_{\pi}^{(0)}, \cdots, \lambda_{\pi}^{(M-1)}\right)$, where

$$
\lambda_{\pi}^{(u)}=E_{\pi^{(u)}}\left\{\left|\beta_{(u)}\right|^{2}\right\} / \sum_{u=0}^{M-1} E_{\pi^{(u)}}\left\{\left|\beta_{(u)}\right|^{2}\right\}, \quad \text { for all } u=0, \cdots, M-1 .
$$

Proof. The risk function under $\left(\xi^{*}, \lambda\right)$ and $\pi$ is

$$
\begin{aligned}
R\left(\xi^{*}, \lambda ; \pi\right)=\left(\sigma^{2}+\sum_{u=0}^{M-1} E_{\pi(u)}\left\{\left|\beta_{(u)}\right|^{2}\right\}\right. & \sum_{u=0}^{M-1} \lambda_{u}^{2} \\
& -\sum_{u=0}^{M-1}\left(2 \lambda_{u}-1\right) E_{\pi(u)}\left\{\left|\beta_{(u)}\right|^{2}\right\} .
\end{aligned}
$$

It is easily verified that $\lambda_{\pi}^{(u)}(u=0, \cdots, M-1)$, given by (5.3), minimize (5.4) under the constraint $\sum_{u=0}^{M-1} \lambda_{u}=1$.

Let $R_{\pi}^{(u)}=E_{\pi(u)}\left\{\left|\beta_{(u)}\right|^{2}\right\}(u=0, \cdots, M-1)$ and $R_{\pi}=\sum_{u=0}^{M-1} R_{\pi}^{(u)}$; then the Bayes risk with respect to an a-priori distribution $\pi$ is

$$
\begin{aligned}
& R\left(\xi^{*}, \lambda_{\pi} ; \pi\right)=\left(\sigma^{2}+R_{\pi}\right) \sum_{u=0}^{M-1}\left(\lambda_{\pi}^{(u)}\right)^{2} \\
& -\sum_{u=0}^{M-1}\left(2 \lambda_{\pi}^{(u)}-1\right) R_{\pi}^{(u)}=R_{\pi}-R_{\pi}^{-1}\left(1-\sigma^{2} / R_{\pi}\right) \sum_{u=0}^{M-1}\left(R_{\pi}^{(u)}\right)^{2} .
\end{aligned}
$$

In particular, when all $\left|\beta_{(u)}\right|^{2}(u=0, \cdots, M-1)$ have the same a-priori distribution, with $R_{\pi}^{(u)}=R_{\pi}^{*}$ for all $u=0, \cdots, M-1$, then the Bayes g.l.s.e. 
is represented by $\lambda^{*}=\left(M^{-1}, M^{-1}, \cdots, M^{-1}\right)$ with a Bayes risk

$$
R\left(\xi^{*}, \lambda^{*}, \pi\right)=\sigma^{2} / M+(M-1) R_{\pi}^{*} .
$$

Theorem 5. $\lambda^{*}=M^{-1} 1^{(M)}$ represents the minimax and admissible g.l.s.e. under randomization procedure $\xi^{*}$ relative to the class of all a-priori distributions $\pi$, such that $R_{\pi}=\sum_{u=0}^{M-1} R_{\pi}^{(u)}=$ const. and $\left(\sigma^{2}<R_{\pi}<\infty\right)$. The minimax risk is given by $(5.6)$

Proof. The minimax risk is the maximal Bayes risk, with respect to all the a-priori distributions $\pi$ in the class considered. The Bayes risk for any of these $\pi$ 's is given by (5.5) where $R_{\pi}=\sum_{u=0}^{M-1} R_{\pi}^{(u)}$ is a given constant. Set the Lagrangian

$$
\begin{array}{r}
L\left(R_{\pi}^{(0)}, \cdots, R_{\pi}^{(M-1)} ; \rho\right)=R_{\pi}-R_{\pi}^{-1}\left(1-\sigma^{2} / R_{\pi}\right) \sum_{u=0}^{M-1}\left(R_{\pi}^{(u)}\right)^{2} \\
+\rho\left(R_{\pi}-\sum_{u=0}^{M-1} R_{\pi}^{(u)}\right) .
\end{array}
$$

By differentiating partially with respect to $R_{\pi}^{(u)}(u=0, \cdots, M-1)$ and $\rho$ and equating the derivatives to zero we arrive at the system of linear equations:

$$
\begin{aligned}
-\left(2 / R_{\pi}\right)\left(1-\sigma^{2} / R_{\pi}\right) R_{\pi}^{(u)}=\rho \text { for all } u= & 0, \cdots, M-1, \\
& \sum_{u=0}^{M-1} R_{\pi}^{(u)}=R_{\pi} .
\end{aligned}
$$

The solution of this system of linear equations is given by $R_{\pi}^{(u)}=R_{\pi} / M$ for every $u=0, \cdots, M-1$. Furthermore, since $R_{\pi} \geqq \sigma^{2}$, all the second order partial derivatives with respect to $R_{\pi}^{(u)}$ are negative. Thus all a-priori distributions $\pi$ such that $R_{\pi}^{(u)}=R_{\pi} / M$ for every $u=0, \cdots, M-1$ are minimax strategies for Nature. As mentioned before, $\lambda^{*}=M^{-1} 1^{(M)}$ is then the unique minimax strategy for the Statistician. The Bayes risk corresponding to $\lambda^{*}$ is given by (5.6). The admissibility of $\lambda^{*}$, relative to the class of a-priori distributions considered, follows from the fact that it is the unique minimax strategy.

6. Estimable linear functions of $\beta$, having uniformly minimum variance g.l.s.e. Let $Y=A x$ be a consistent system of linear equations. If $x=A^{-} y$ is any solution to this system then $A^{-}$is called a generalized inverse of $A$ (see [1]). Rao [8] proves the following theorem: If a linear function $\zeta^{\prime} \beta$ is such that for a given generalized inverse of $\left(C_{v}\right)^{\prime}\left(C_{v}\right)$, say $\left[\left(C_{v}\right)^{\prime}\left(C_{v}\right)\right]^{-}$, the equality

$$
\zeta^{\prime}\left[\left(C_{v}\right)^{\prime}\left(C_{v}\right)\right]^{-}\left[\left(C_{v}\right)^{\prime}\left(C_{v}\right)\right]=\zeta^{\prime}
$$

holds, for some $v=0, \cdots, M-1$, then the value of $\zeta^{\prime} \beta=\zeta^{\prime}\left[\left(C_{v}\right)^{\prime}\left(C_{v}\right)\right]^{-}$ $\left(C_{v}\right)^{\prime} Y\left(X_{v}\right)$ is unique; and the function $\zeta^{\prime} \beta$ is estimable by $\zeta^{\prime} \hat{\beta}$; i.e., $E\left(\zeta^{\prime} \hat{\beta}\right)=$ $\zeta^{\prime} \beta$. Moreover, if $\zeta^{\prime} \tilde{\beta}$ is any unbiased estimator of $\zeta^{\prime} \beta$ then, $\operatorname{Var}\left\{\zeta^{\prime} \hat{\beta}\right\} \leqq \operatorname{Var}\left\{\zeta^{\prime} \beta\right\}$. This theorem is applied to characterize the class of all estimable linear functions of $\beta$ having a uniformly minimum variance. 
From the definition of a generalized inverse of a matrix, and according to (3.2), if $\left[\left(C_{v}\right)^{\prime}\left(C_{v}\right)\right]^{-}$is any generalized inverse of $\left(C_{v}\right)^{\prime}\left(C_{v}\right)$ then

$$
\hat{\beta}_{v}=\left[\left(C_{v}\right)^{\prime}\left(C_{v}\right)\right]^{-}\left(C_{v}\right)^{\prime} Y\left(X_{v}\right)
$$

is a g.l.s.e. of $\beta$. Comparing (6.2) with (3.7) we conclude that every generalized inverse of $\left(C_{v}\right)^{\prime}\left(C_{v}\right)$, in $a N=s^{m}$ factorial system, is given by,

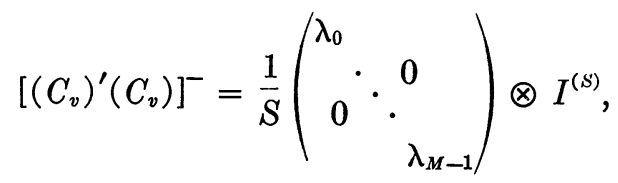

for all $v=0, \cdots, M-1$; where $\sum_{u=0}^{M-1} \lambda_{u}=1$. According to $(3.3)\left(C_{v}\right)^{\prime}\left(C_{v}\right)=$ $S\left[\left(1, c_{v 1}^{(M)}, \cdots, c_{v(M-1)}^{(M)}\right)^{\prime}\left(1, c_{v 1}^{(M)}, \cdots, c_{v(M-1)}^{(M)}\right)\right] \otimes I^{(S)}$. Thus,

$$
\begin{aligned}
& {\left[\left(C_{v}\right)^{\prime}\left(C_{v}\right)\right]^{-}\left[\left(C_{v}\right)^{\prime}\left(C_{v}\right)\right]} \\
& =\left(\lambda_{0} c_{v 0}^{(M)}, \lambda_{1} c_{v 1}^{(M)}, \cdots, \lambda_{M=1} c_{v(M-1)}^{(M)}\right)^{\prime}\left(c_{v 0}^{(M)}, c_{v 1}^{(M)}, \cdots, c_{v(M-1)}^{(M)}\right) \otimes I^{(S)}
\end{aligned}
$$

where $\sum_{u=0}^{M-1} \lambda_{u}=1$.

Let $\zeta^{\prime}=\left(\zeta_{(0)}, \zeta_{(1)}, \cdots, \zeta_{(M-1)}\right)$ be any $N$-dimensional vector, where $\zeta_{(u)}(u=0, \cdots, M-1)$ are $S$-dimensional subvectors of $\zeta$. Then for every $v=0, \cdots, M-1$, we obtain:

$$
\zeta^{\prime}\left[\left(C_{v}\right)^{\prime}\left(C_{v}\right)\right]^{-}\left[\left(C_{v}\right)^{\prime}\left(C_{v}\right)\right]=\left(\eta_{(0)}^{\prime}(v), \cdots, \eta_{(M-1)}^{\prime}(v)\right)^{\prime}
$$

where

$$
\eta_{(u)}^{\prime}(v)=c_{v u}^{(M)} \sum_{w=0}^{M-1} \lambda_{w} c_{v w}^{(M)} \zeta_{(w)}^{\prime}, \quad \text { for all } u=0, \cdots, M-1 .
$$

Hence, $\zeta^{\prime}\left[\left(C_{v}\right)^{\prime}\left(C_{v}\right)\right]^{-}\left[\left(C_{v}\right)^{\prime}\left(C_{v}\right)\right]=\zeta^{\prime}$ if, and only if,

$$
\zeta_{(u)}^{\prime}=c_{v u}^{(M)} \sum_{w=0}^{M-1} \lambda_{w} c_{v w}^{(M)} \zeta_{(w)}^{\prime}, \quad \text { for all } u=0, \cdots, M-1 .
$$

Thus, the set of all $N$-dimensional functionals $\zeta$ which yield minimum variance unbiased linear estimators $\zeta^{\prime} \hat{\beta}$ of the linear functions $\zeta^{\prime} \beta$ uniformly in $\beta$ is

$$
\begin{aligned}
& \left\{\zeta: \zeta^{\prime}=\left(\zeta_{(0)}^{\prime}, \cdots, \zeta_{(M-1)}^{\prime}\right) ;\right. \\
& \left.\zeta_{(u)}^{\prime}=c_{v u}^{(M)} \sum_{w=0}^{M-1} \lambda_{w} c_{v w}^{(M)} \zeta_{(w)}^{\prime} ; \text { and } \sum_{w=0}^{M-1} \lambda_{w}=1\right\}
\end{aligned}
$$

where $\left(\lambda_{0}, \cdots, \lambda_{M-1}\right)$ represents the g.l.s.e. $\hat{\beta}$. Given a linear function $\zeta^{\prime} \beta$ we should check whether there exists a matrix $\left(C_{v}\right)(v=0, \cdots, M-1)$ and a vector $\left(\lambda_{0}, \cdots, \lambda_{M-1}\right)$ for which $(6.7)$ holds. For example, if we want to estimate the sum of the components of $\beta$, i.e., $I^{(N) \prime} \beta$ then, take $v=M-1$ (i.e., perform the experiment on the block $X_{M-1}$ ) and the g.l.s.e. represented by $\lambda^{*}=M^{-1} 1^{(M)}$. Then, since $c_{(M-1) u}^{(M)}=1$ for all $u=0, \cdots, M-1$, Condition (6.7) holds. That is, the linear function $1^{(M) \prime} \beta$ has a uniformly minimum var- 
iance g.l.s.e. On the other hand, there are many linear functions of $\beta$ which are unestimable by a fixed fractional replication procedure. For example, the linear function $\zeta^{\prime} \beta=\beta_{1}$ is not estimable by a fixed fractional replication procedure. It is estimable by a randomization procedure $\xi^{*}$ and g.l.s.e. represented by $\lambda=(1,0, \cdots, 0)$, but its variance depends on $\beta$ (see Zacks [13]). A locally minimum variance g.l.s.e. of $\beta_{1}$ can be attained (see Takeuchi [11]) but not a uniformly minimum variance estimator.

Acknowledgment. The author gratefully acknowledges Professors H. Solomon and $\mathrm{H}$. Chernoff for their helpful comments and encouragement.

\section{REFERENCES}

[1] Ben-Israel, A. and Wersan, S. J. (1962). A least-squares method for computing the generalized-inverse of an arbitrary complex matrix. O.N.R. Research Memorandum No. 61. Systems Research Group, Northwestern Univ.

[2] Dempster, A. P. (1961). Random allocation designs I: On general classes of estimation methods. Ann. Math. Statist. 31 885-905.

[3] Dempster, A. P. (1961). Random allocation designs II: Approximative theory for simple random allocations. Ann. Math. Statist. 32 387-405.

[4] Ehrenfeld, S. and Zacks, S. (1961). Randomization and factorial experiments. Ann. Math. Statist. 32 270-297.

[5] Ehrenfeld, S. and Zacks, S. (1963). Optimum strategies in factorial experiments. Ann. Math. Statist. 34 780-791.

[6] Kempthorne, O. (1952). The Design and Analysis of Experiments. Wiley, New York.

[7] Kurkjian, B. and Zelen, M. (1962). A calculus for factorial arrangements. Ann. Math. Statist. 33 600-619.

[8] Rao, C. R. (1962). A note on a generalized inverse. J. Roy. Statist. Soc. B 24152.

[9] Sнан, B. V. and Kempthorne, O. (1962). Some properties of random allocation designs. Technical Report, Statist. Lab. Iowa State Univ.

[10] Sнан, B. V. and Kempthorne, O. (1962). Randomization in fractional factorial. Technical Report, Statist. Lab. Iowa State Univ.

[11] TaKeUChI, K. (1961). On a special class of regression problems and its applications: Random combined fractional factorial designs. Rep. Stat. Appl. Res. JUSE, 7 $1-33$.

[12] TaKeUChI, K. (1961). On a special class of regression problems and its applications: Some remarks about general models, Rep. Statist. Appl. Res. Un. Japan Sci. Engrs. 8 7-17.

[13] ZaCKs, S. (1963). On a complete class of linear unbiased estimators for randomized factorial experiments. Ann. Math. Statist. 34 769-779. 